\title{
Microchip Technologies for the Analysis of Biological Cells
}

\author{
Takanori Ichiki, Satomi Shinbashi, Takekazu Ujiie, and Yasuhiro Horiike \\ Department of Electrical and Electronics Engineering, Toyo University, \\ 2100 Kujirai, Kawagoe 350-8585, Japan \\ ${ }^{\ddagger}$ Department of Materials Science, The University of Tokyo, \\ 7-3-1 Hongo, Bunkyo-ku, 113-8656, Japan \\ ichiki@eng.toyo.ac.jp
}

\begin{abstract}
In the post-genome era, the importance of cell analysis is increasingly appreciated since the cell is the minimum unit of the life phenomena. On-chip manipulation and measurement of biological cells are recognized to be an important subject for the establishment of novel analytical tools and methodologies for the forthcoming new cell biology. In this paper on-chip manipulation of biological cells and microparticles by means of dielectrophoresis and on-chip detection of single biological cells by ac impedance spectrometry have been investigated using microfulidics devices with embedded microelectrodes which were fabricated by LSI compatible microfabrication technologies.
\end{abstract}

Keywords: microchip, microelectrode, biological cell, dielectrophoretic manipulation, single-cell impedance measurement

\section{Introduction}

Development of micro total analysis systems $(\mu \mathrm{TAS})$, which enable high-speed separation analysis of tiny amounts of substance, has progressed rapidly [1]. By applying semiconductor microfabrication technology, microfluidics devices with several tens $\mu \mathrm{m}$ wide channels have been fabricated on glass or plastic substrates. Representative successful devices are miniaturized flow injection analysis (FIA) systems such as gas chromatography (GC) and microcapillary electrophoresis $(\mu \mathrm{CE})$, and are utilized in combination with on-chip detection methods, such as laser induced fluorescence (LIF) detection, electrochemical measurement and so on. Research of $\mu$ TAS started in the early 90s [2-4], and was drastically accelerated from the late 90 s when much attention was attracted to $\mu \mathrm{CAE}$ (microfabricated capillary array electrophoresis) devices which aimed at high throughput DNA analysis [5-7]. Still now, in the post genome era, $\mu$ TAS research continues rapid growth both qualitatively and quantitatively, expanding an object field further. Research and development which target the field of bioinformatics such as single nucleotide polymorphisms (SNPs) analysis, proteome analysis, etc. are prosperous for the present. On the other hand, one of the most important subject in the future bioinformatics research is the biological phenomena of the cell, which is the basic unit of a living matter, and the behavior of biomolecules inside it. As for the measurement of biological cells, unlike DNA sequence analysis, much room for technical development is still left behind. As described in the famous textbook, "what we can learn from cells depends on the tools at our disposal, and major advances in cell biology have frequently sprung from the introduction of new techniques [8]". There are great hopes that innovative analytical techniques for cellomics might appear by the introduction of the microchip technology. Besides the application in bioinformatics, on-chip manipulation and test of blood cells are important subject for the development of medical point of care testing (POCT) equipments. Practical technologies are required for the on-chip pretreatment of whole blood, such as separation and concentration of blood cells. In this paper the manipulation of biological cells by means of dielectrophoresis (DEP) [9] and the on-chip measurement of single biological cells by impedance spec- 
trometry have been investigated using microfulidics chips with embedded microelectrodes fabricated by LSI compatible microfabrication technologies.

\section{Experimental}

2.1. Fabrication of Microfluidics Devices with Embedded Microelectrodes

The chip fabrication process as depicted in Fig. 1 has been developed so that platinum interdigitated array (IDT) electrodes may be completely embedded into the inner wall of the microcapillary and no steric obstacle may arise in the microchannels. A chrome film of $100 \mathrm{~nm}$ thickness was sputter-deposited on an optically flat quartz substrate of $0.5 \mathrm{~mm}$ thickness and 20 by $20 \mathrm{~mm}$ size, and were patterned using electron beam (EB) direct lithography and highly precise chrome dry etching [10]. Using this metal mask, the 200-nm-deep electrode patterns were etched into the quartz substrate in fluorine-based inductively coupled plasmas as shown in the upper photo of Fig. 2. After removal of chrome residue and wet chemical cleaning, a chrome film of $100 \mathrm{~nm}$ thickness was deposited again on an etched quartz substrate as an adhesive layer of the platinum electrode. In the next step, photoresist was spin-coated over the substrate, and then etched back to expose the surface of the chrome film deposited outside the electrode pattern, which was subsequently removed by wet chemical etching. Microelectrodes were accomplished by the deposition of 100-nm-thick platinum films using EB evaporation and lift-off as shown in the lower photo of Fig. 2. In the final step, the quartz substrate with microelectrodes was press-

1. Cr film deposition

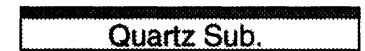

2. EB direct lithography \& Dry etching of $\mathrm{Cr}$

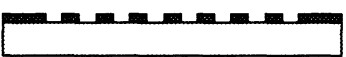

3. ICP etching of quartz บับบันทั

4. Removal of residual $\mathrm{Cr}$ mask

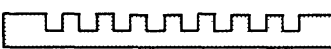

5. Deposition of $\mathrm{Cr}$ binder layer 口ичйла

6. Spin coating of photoresist

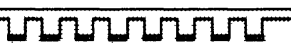

Fig. 1 Fabrication process of microfluidics chips with embedded platinum microelectrodes.

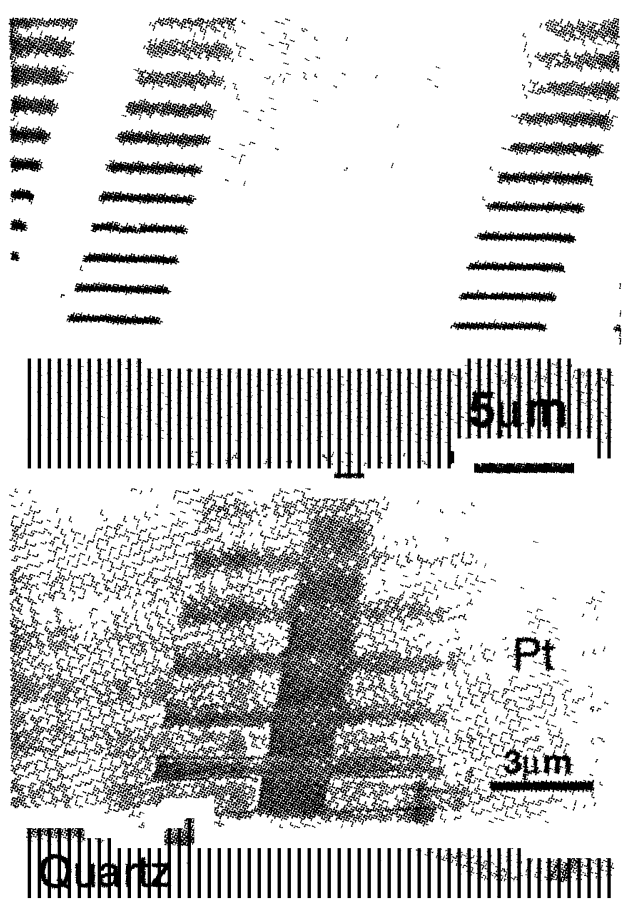

Fig. 2. SEM photos of microelectrode patterns engraved on a quartz substrate (upper) and those completely embedded with platinum(lower).

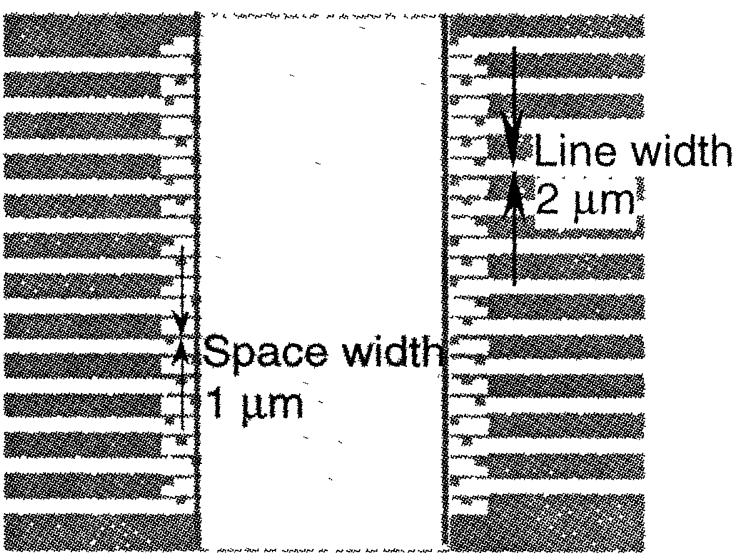

Fig. 3. Typical layout of Pt microelectrodes designed for DEP manipulation experiments.

bonded together with the other quartz substrate on which a microcapillary of a few tens $\mu \mathrm{m}$ depth and width was engraved by plasma etching and two reservoir holes were opened by ultrasonic machining [11]. Figure 3 shows the typical layout of platinum microelectrodes embedded in a microcapillary chip.

\subsection{Cell Manipulation Using Dielectrophoresis}

Cell manipulation using DEP was examined in order to realize trapping and filtering of cells flowing inside the microcapillary formed on a glass chip. "Dielectrophoresis" is the term that describes the translational motion of uncharged dielectric particles in spatially nonuniform electric fields due to the di- 
electric force [12-14]. The dielectric force which acts on a dielectric sphere placed in the nonuniform electric field is proportional to the volume of the particle and the gradient of the square of the local electric field strength. In addition, the DEP force is distinguished between positive DEP, which attracts particles toward the electrode, and negative DEP, which repels particles, depending on the complex permittivity of the particle and the surrounding medium [15].

Sheep red blood cells (ShE; sheep erythrocyte) separated from the sheep whole blood (Nippon BioTest Laboratories Inc., Japan) or styrene beads of 1.00 $\mu \mathrm{m}$ diameter (1.0 STADEX SC-101-S; JSR Corp., Japan) and $3.25 \mu \mathrm{m}$ diameter (Dynospheres SS-031$\mathrm{R}$; JSR Corp., Japan), which are frequently used as size standard particles, were suspended in $\mathrm{pH} 7.4$ phosphate buffered saline (PBS) and introduced into the injection reservoir of the prototype chip whose microchannel was filled with the buffer solution beforehand. Then the chip was installed on an optical microscope (Model TE200; Nikon, Japan) equipped with a CCD camera (Model CS5270; Tokyo Electronic Industry Co., Ltd., Japan). A function generator (Model 1920A; NF Electronic Instruments, Japan) was connected to the terminal electrodes on a chip to supply a sinusoidal voltage with tuned frequency and amplitude. The water level of reservoirs was adjusted in order to control the velocity of particle transportation owing to the hydropressure flow inside the capillary. The behavior of particles at the time of being applied the ac voltage to the microelectrodes was observed and recorded on the personal computer.

\subsection{AC Impedance Spectroscopy}

Electrical measurement of single cells was attempted with a viewpoint of using electric impedance as a physical marker for the typing of biological cells [16-18]. In the preliminary experiment, inner wall coating of the microcapillary chip with polyethrene glycol (PEG) or gelatin was examined since adsorption of serum proteins and blood cells on the surfaces of electrodes and capillary inner walls are anticipated to degrade the reproducibility of measurements. The procedure of PEG coating is as follows. Prior to coating, a microcapillary chip was cleaned by sequential exposure to $1 \% \mathrm{NaOH}$ $\left(60^{\circ} \mathrm{C}\right)$ and $3 \% \mathrm{HCl}$ for 10 minutes, and then rinsed repeatedly with distilled water. The chip was dried in a vacuum chamber $(20 \mathrm{~Pa})$ and then coated with PEG of $15000-20000$ Da via 6 hour, $65^{\circ} \mathrm{C}$ exposure to a $10 \%$ PEG solution in $0.05 \mathrm{M}$, sodium phosphate at $\mathrm{pH} 7.3$, followed by rinsing with water. On the other hand, a thin gelatin coating was formed in the following procedure. The gelatin was added to $150 \mathrm{mM} \mathrm{pH} 7.4$ veronal buffer at low concentration of $0.1 \%$. The chip was flushed with this gelatin veronal buffer (GVB), and then rinsed repeatedly with distilled water. A thin gelatin layer was formed on the inner wall of the microcapillary by adsorption from the buffer solution. After introducing diluted serum or blood cells in a microchannel, complex impedance between IDT microelectrodes was measured by supplying ac voltages by FRA (Frequency Response Analyzer: Solatron SI1260, UK.) as shown in Fig. 4.

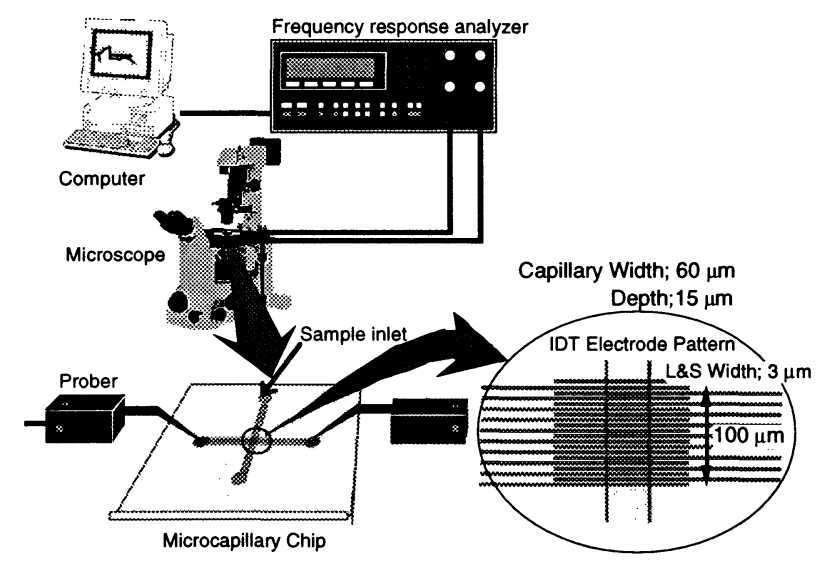

Fig. 4. The setup for on-chip electrical impedance measurement of biological cells..

\section{Results and Discussion}

3.1. Cell Manipulation Using Dielectrophoresis

At the first place, dielectrophoretic trapping of biological cells which flow inside the microfluidics device was attempted using sheep red blood cells suspended in phosphate buffer saline (PBS) at $1 \times 10^{8} /$ $\mathrm{ml}$. Successful trapping between castellated IDT electrodes was observed when the ac voltage at the frequency of $600 \mathrm{kHz}$ and the amplitude of $25 \mathrm{~V}$, corresponding to the electric field strength $2.7 \mathrm{MV} / \mathrm{m}$, was applied.

Secondly, size separation of flowing particles was attempted utilizing the fact that the DEP force is proportional to the particle volume. The mixture of styrene beads with two different diameters of $1 \mu \mathrm{m}$ and $3.25 \mu \mathrm{m}$ was used as a model sample. Figure 5 shows the flow velocity of styrene beads under the alternative electric field at $600 \mathrm{~Hz}$ as a function of the applied peak-to-peak voltage. The migration velocity of styrene beads of both sizes decreased with the increase of the applied voltage, and above $7 \mathrm{~V}$ only the velocity of styrene beads with $3.25 \mu \mathrm{m}$ diameter became zero. Namely all the styrene beads with the diameter of $3.25 \mu \mathrm{m}$ were trapped, while the styrene beads of $1 \mu \mathrm{m}$ diameter flew toward a waste reser- 
voir.

In addition, another application of DEP trapping was demonstrated using $3.25 \mu \mathrm{m}$ diam. styrene beads diluted at $10^{10} / \mathrm{ml}$ with $\mathrm{pH} 9.0$ Tris buffer solution. They were injected into the microchannel at the flow rate of $50 \mu \mathrm{m} / \mathrm{s}$. Just at the moment of applying high frequency voltage at $600 \mathrm{kHz}$ and $10 \mathrm{~V}$ to a local pair of interdigitated electrodes, styrene beads were trapped in line, and then they started to flow again after turning off the voltage as shown in Fig. 6. Such

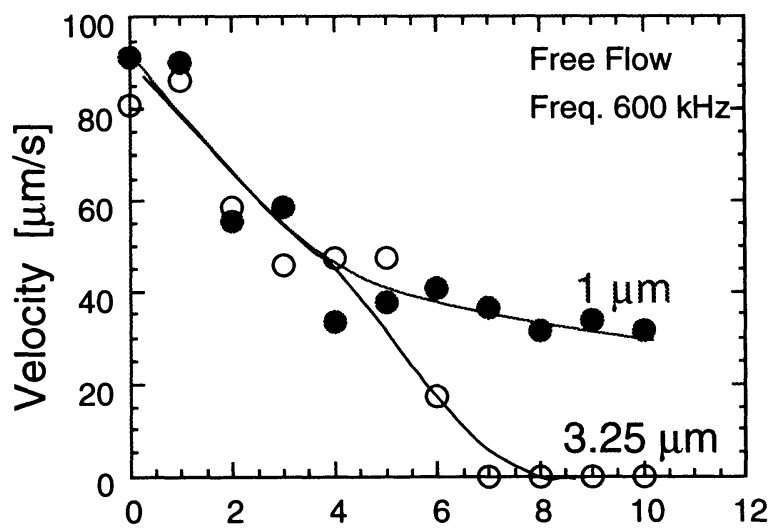

\section{Applied Peak-to-Peak Voltage [V]}

Fig. 5. Flow velocity of $1 \mu \mathrm{m}$ and $3.25 \mu \mathrm{m}$ diam. styrene beads under the DEP force as a function of the applied peak-to-peak voltage.

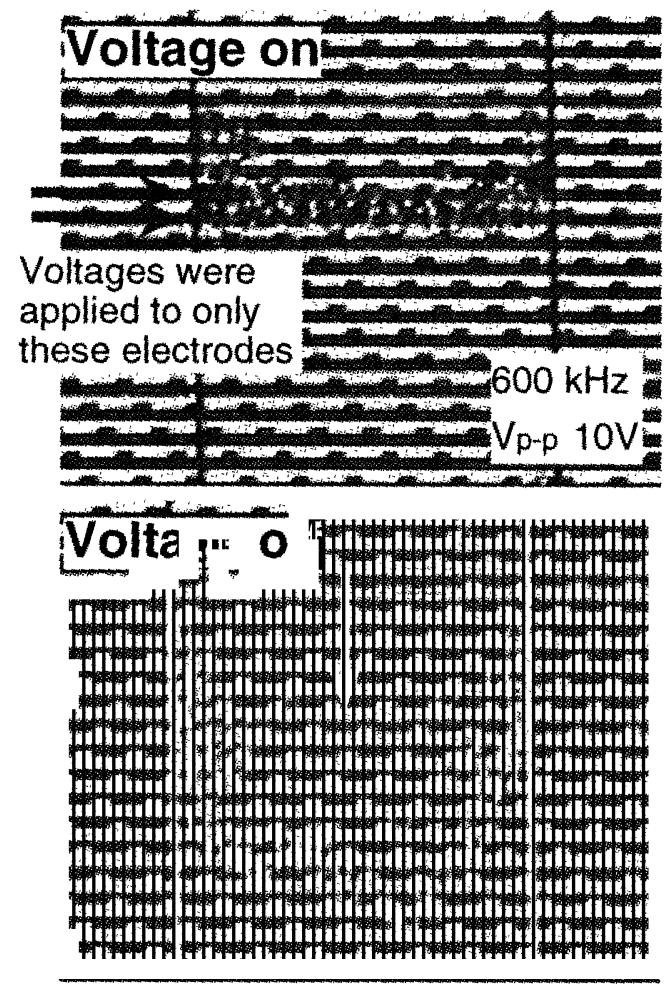

Fig. 6. Dielectrophoretic trapping of styrene beads in liquid-flow on a microcapillrary chip with embedded microelectrodes.. behaviors of microparticels are available for the sample concentration.

\subsection{AC Impedance Spectroscopy}

For the purpose of examining whether PEG coating is accomplished on platinum electrodes as well as on a quartz substrate, surface state of quartz and platinum substrates after PEG coating was compared using $\mathrm{x}$-ray photoelectron spectroscopy (XPS). PEG coating was performed in the same procedure on both substrates. As shown in Fig. 7, almost the same $C_{1 s}$ peak intensity was detected from both quartz and platinum substrates. Therefore the PEG is considered to be coated also on platinum electrodes as well as on the quartz surface. Subsequently, in order to check the adhesion control of the inner wall of the capillary, the diluted serum protein was introduced into the microcapillary chip, and the reproducibility of impedance measurements was checked. Figure 8 shows the typical results of repeated measurements of two solutions of (a) serum
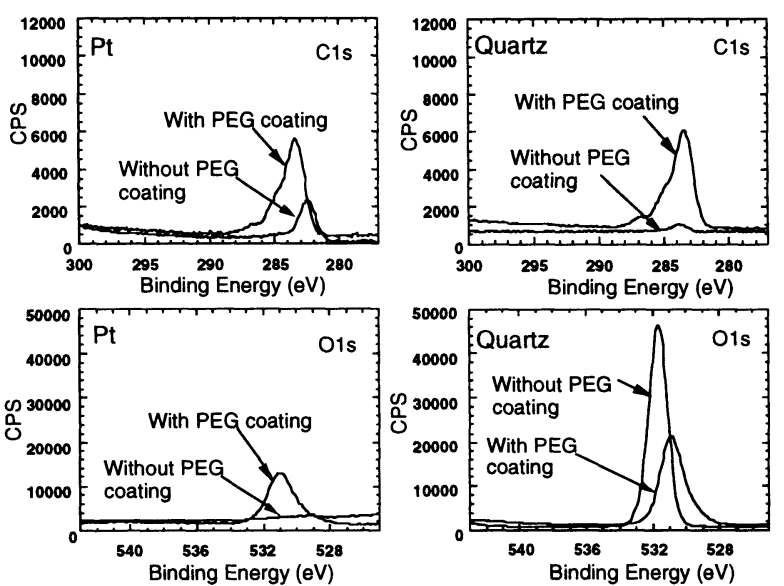

Fig. 7. $\mathrm{C}_{1 \mathrm{~s}}$ and $\mathrm{O}_{1 \mathrm{~s}} \mathrm{XPS}$ spectra measured from platinum and qurtz substrates with/without PEG coatings.

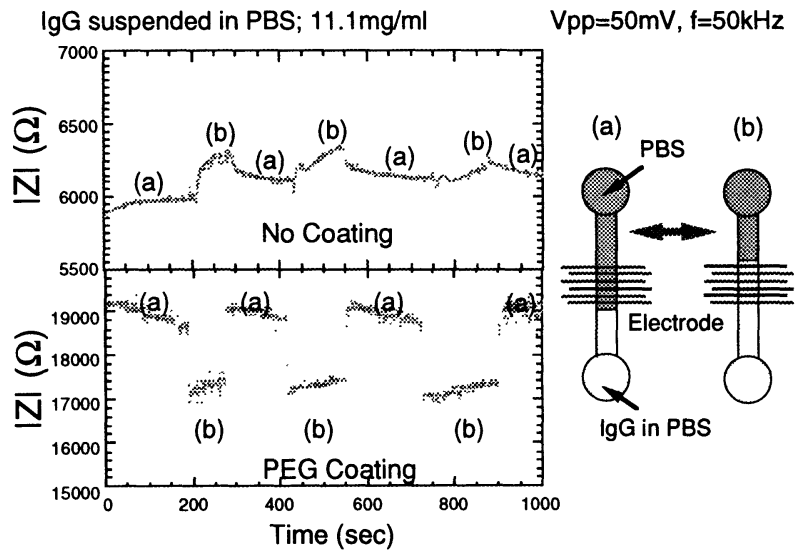

Fig. 8. Impedance values of diluted IgG samples measured on chips with/without PEG coatings. 
protein IgG and (b) phosphate buffer solution (PBS). The impedance between a pair of electrodes with and without PEG coating was measured at 50 $\mathrm{kHz}$. It is apparent that the reproducibility of impedance measurement is improved by PEG coating since nonspecific adsorption of proteins on the electrode surface was suppressed by the brush structure of PEG polymer.

Another preliminary experiment was the impedance spectra measurement of stationary sheep red blood cells filled in the microchannel. Measurements of the PBS solution without red blood cells were carried out as a control experiment. Impedance spectra obtained by applying two different voltage amplitudes of 10 and $100 \mathrm{mV}$ are exhibited in Fig. 9. It revealed the specific impedance structure in the frequency range of $100 \mathrm{kHz}-10 \mathrm{MHz}$. This structure dominantly originates from the capacity of cell membranes, and is called $\beta$ dispersion due to Maxwell-Wagner effect [19].

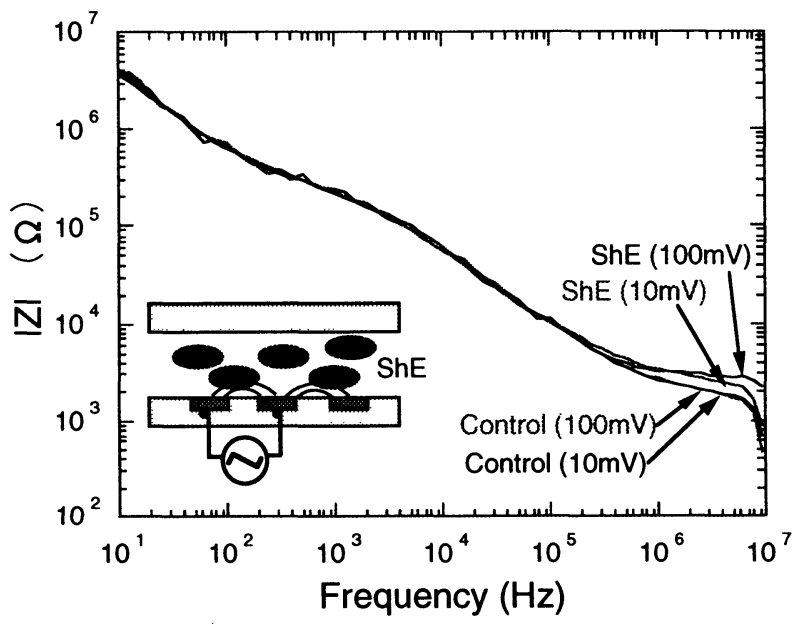

Fig. 9. Impedance spectra of sheep red blood cells.. Amplitudes of the applied ac voltages were 10 or $100 \mathrm{mV}$. Control measurements were carried out for PBS solutions without mixing sheep red blood cells.

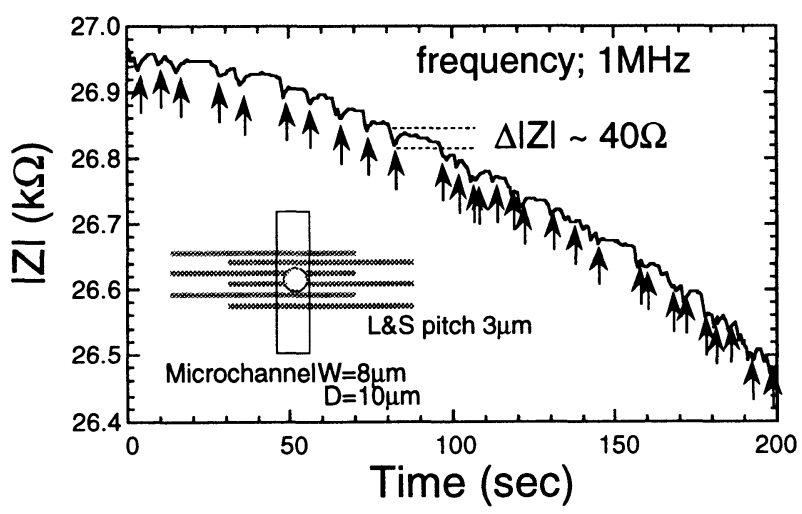

Fig. 10. Detection of the flowing single sheep red blood cells by the ac impedance measurement. The impedance peaks ascribed to single cells are indicated by each arrow.
Based on above results, detection of single red blood cells that passes over the electrode was attempted by the real-time impedance measurement. The chip used in this experiment had a microchannel of $8 \mu \mathrm{m}$ width and $10 \mu \mathrm{m}$ depth, which is the size of the same order with sheep red blood cells $(3 \mu \mathrm{m})$, and only three pairs of IDT electrodes so as to attain high detection sensitivity and precise position tracking. Prior to the impedance measurement experiment, PEG coating was carried out. Impedance measurement was carried out at fixed frequencies at $100 \mathrm{kHz}, 500 \mathrm{kHz}$ and $1 \mathrm{MHz}$. Although no significant signal change was observed at $100 \mathrm{kHz}$ and 500 $\mathrm{kHz}$, single cells flowing over the microelectrodes were successfully detected at the frequency of 1 $\mathrm{MHz}$ via the impedance difference of $40 \Omega$ as shown in Fig. 10. It should be mentioned that the dielectrophoretic force that attracts sheep red blood cells toward the electrode makes an important contribution for the high sensitivity detection.

\section{Conclusion}

On-chip manipulation and measurements of biological cells were studied using a microcapillary chip with completely embedded platinum microelectrodes. DEP force is demonstrated to be available for trapping, concentration and size filtering of cells and microparticles. Successful detection of single blood cells flowing inside the microcapillary on a chip was carried out by ac impedance measurement at $1 \mathrm{MHz}$. The electrical impedance measurement using microelectrodes enables the accurate position tracking and typing of single cells and will be available as the detection module for the single-cell level sorter device that has been developed recently [20].

\section{Acknowledgments}

This work was supported by a Grant-in-Aid for Scientific Research from the Japan Society for the Promotion of Science, and the High-Tech Research Center organized by the Ministry of Education, Sports and Culture, Japan.

\section{References}

1. For example, Microsystem Technology in Chemistry and Life Science, A. Manz and H. Becker, Eds., Springer-Verlag, Berlin, 1998.

2. A. Manz, N. Graber, and H. M. Widmer, Sens. Actuator B, 1 (1990) 244.

3. A. Manz, D. J. Harrison, E. M. J. Verpoorte, J. C. Fettinger, A. Paulus, H. Ludi, and H. M. Widmer, J. Chromatogr., 593 (1992) 253.

4. D. J. Harrison, K. Fluri, K. Seiler, Z. H. Fan, C. S. 
Effenhauser, and A. Manz, Science, 261 (1993) 859. 5. A. T. Woolley, G. F. Sensabaugh, and R. A. Mathies, Anal. Chem., 69 (1997) 2181.

6. Y. N. Shi, P. C. Simpson, J. R. Scherer, D. Wexler, C. Skibola, M. T. Smith, and R. A. Mathies, Anal. Chem., 71 (1999) 5354.

7. I. L. Medintz, B. M. Paegel, R. G. Blazej, C. A. Emrich, L. Berti, J. R. Scherer, and R. A. Mathies, Electrophoresis, 22 (2001) 3845.

8. Molecular biology of the cell, 3rd ed. by B. Alberts, D. Bray, J. Lewis, M. Raff, K. Roberts, and J.D. Watson, Garland Publishing, New York, 1994.

9. T. Ujiie, A. Yamazaki, M. Watanabe, T. Okuda, T. Ichiki and Y. Horiike, Digest of papers 2000 Int. Microprocesses and Nanotechnology Conf. Tokyo, Japan, July 11-13 (2000) 28.

10. T. Ichiki, S. Takayanagi and Y. Horiike, J. Electrochem. Soc., 147 (2000) 4289.

11. T. Ujiie, T. Kikuchi, T. Ichiki and Y. Horiike, Jpn. J. Appl. Phys., 39 (2000) 3677.

12. H. A. Paul, Dielectrophoresis, Cambridge University Press, Cambridge (1978).
13. M. Washizu, S. Suzuki, O. Kurosawa, T. Nishizaka, and T. Shinohara, IEEE Trans. Industrial Applications, 30 (1994) 835.

14. R. Pethig, Y. Huang, X-B. Wang, and J. P H Burt, J. Phys. D, 24 (1992) 881.

15. T. Müller, A. Gerardino, T. Schnelle, S. G. Shirley, F. Bordoni, G. D. Gasperis, R. Leoni and G. Fuhr, J. Phys. D, 29 (1996) 340.

16. H. E. Ayliffe, A. B. Frazier and R. D. Rabbitt, IEEE J. Microelectromech. Syst., 8 (1999) 50.

17. C. K. Fuller, J. Hamilton, H. Ackler and P. R. C. Gascoyne, Micro Total Analysis Systems 2000, A.van den Berg, W. Olthusis and Bergveld Eds., Kluwer Academic Publishers, Netherland (2000) p 265.

18. S. Gawad, L. Schild and Ph. Renaud, Lab on a Chip, 1 (2001) 76.

19. R. Pethig and D. B. Kell, Phys. Med. Biolo., 32 (1987) 933.

20. T. Ichiki, T. Ujiie, T. Hara, Y. Horiike and K. Yasuda, Micro Total Analysis Systems 2001, J. M. Ramsey and A.van den Berg, Eds., Kluwer Academic Publishers, Netherland (2001) p 271. 